\title{
Comportamiento de las arcillas del Área Metropolitana de Cúcuta sometidas a proceso de moldeo por extrusión.
}

John Freddy Gelves Diaz¹, Jorge Sánchez Molina1 ｜ G. Peña Rodríguez²

Recibido:

2 de mayo de 2009

Aceptado:

30 de noviembre de 2009
${ }^{1}$ Centro de Investigación de Materiales Cerámicos, Grupo de Investigación en Tecnología Cerámica GITEC, Universidad Francisco de Paula Santander, Cúcuta-Colombia. e-mail: johngelves@yahoo.es; j_sanchezmolina@yahoo.es

${ }^{2}$ Grupo de Investigación en Tecnología Cerámica, Departamento de Física Universidad Francisco de Paula Santander, Cúcuta-Colombia. e-mail: ggabrielp@yahoo.com

\section{Resumen}

Se presenta el comportamiento de materiales arcillosos ubicados en la zona Metropolitana de San José de Cúcuta, cuando son sometidos al proceso de conformado por extrusión. La investigación se llevó a cabo, utilizando muestras extraídas de las formaciones geológicas León (municipio Cúcuta) y Guayabo (municipio de El Zulia y Los Patios). Se estudió el efecto de las materias primas (contenido de arena y tamaño de partícula) sobre el proceso de moldeo, determinando el índice de plasticidad, porcentaje de absorción de agua y la resistencia a la flexión. Las dimensiones de las probetas en verde fueron: largo $4 \mathrm{~cm}$, ancho $4 \mathrm{~cm}$ y alto $1 \mathrm{~cm}$. El proceso de secado, se llevo a cabo usando estufa de circulación forzada durante 24 horas a $110^{\circ} \mathrm{C}$, mientras que la cocción se realizó a través de horno eléctrico a una temperatura máxima de $1000{ }^{\circ} \mathrm{C}$ por 6 horas. Se encontró, que el porcentaje de arcilla presente en las muestras de las dos formaciones geológicas es similar ( $21 \%)$, mientras que la concentración de arena y limos variaron considerablemente según la formación geológica (Guayabo y León). El índice de plasticidad (IP) para las muestras, reportó un valor promedio de 24,5\% para la formación Guayabo, y 29.5\% para la León.

La resistencia a la flexión y el porcentaje de absorción de agua no presentó variación significativa. Lo ensayos realizados a las mezclas con variación de material arenoso permitió establecer que los rangos más adecuados para la formulación de las pastas cerámicas usando arcillas del área Metropolitana de San José de Cúcuta, deben estar en: la fracción arenosa entre 20 y 27\%, 23 y 28\% para la fracción de limos y entre 47 y 58\% para la fracción arcillosa; el indice de plasticidad debe estar cercano al 22\%. Las formulaciones establecidas en estos rangos permiten obtener buenas velocidades de extrusión, consumos energéticos adecuados y una buena calidad del producto fabricado.

Palabras clave: Plasticidad, compactación, tamaño de partícula, moldeo, extrusión 


\section{Abstract}

The behaviour of clay materials located in the metropolitan area of San Jose de Cucuta when subjected to extrusion process is presented. The research was conducted using samples from the Leon geological formations (municipality of Cucuta) and Guayabo (municipality of El Zulia and Los Patios). We studied of the effect of raw materials (sand content and particle size) on the molding process, through the plasticity index, water absorption percent and the bending strength. The dimensions of the samples were: length $4 \mathrm{~cm}$, width $4 \mathrm{~cm}$ and 1 $\mathrm{cm}$ high. The drying process was carried out using forced circulation oven for 24 hours at $110^{\circ} \mathrm{C}$, while the baking is make through electric furnace at a maximum temperature of $1000^{\circ} \mathrm{C}$ for 6 hours. It was found that the percentage of clay present in the samples of the two geological formations is similar $(\sim 61 \%)$, while the sand concentration and silt varied considerably according to the geological formation (Guayabo and Leon). We reported the average plasticity index (IP) for samples, being of 24,5\% for Guayabo formation, and 29,5\% for Leon formation. The bending strength and water absorption percent did not present significant change. The tests conducted on mixtures with variation of the sand concentration revealed that the most suitable for the formulation of ceramic pastes using minerals of the metropolitan area of San Jose de Cucuta area must be: the sandy fraction between 20 and 27\%, 23 y 28\% for the fraction of the slime and between 47 and 58\% for the clay fraction, the plasticity index should be close to $22 \%$. The mixtures these ranges can get good results for extrusion speed, adequate energy consumption and good quality of the manufactured product by molding extrusion process.

Keywords: Plasticity, compaction, particle size, molding, extrusion

\section{Introducción}

$\mathrm{L}$ a zona metropolitana de Cúcuta, conformadaporlosmunicipios de Villadel Rosario, Zulia, San Cayetano y Los Patios es un sector muy rico en materiales arcillosos, lo cual ha permitido el establecimiento de un conglomerado de empresas dedicadas a la explotación y transformación de este material. La mayor parte de estas empresas están dedicadas a la fabricación de productos para el sector de la construcción tales como bloques, ladrillos, tejas, pisos y revestimientos, donde generalmente utilizan el proceso de conformado por extrusión en su fabricación [1].
Aunque el sector cerámico regional es reconocido a nivel nacional e internacional, existe un bajo conocimiento de tipo técnico sobre las características de las materias primas y del comportamiento de las mismas durante el proceso de fabricación de las piezas cerámicas. Esta falta de conocimiento, contribuye notoriamente a que se reduzca la calidad del producto terminado, y a que se realicen procesos que implican un sobre costo de la pieza fabricada.

En los últimos años se han realizado varios estudios con el fin de lograr una mejor caracterización del sector cerámico regional, sin embargo la mayoría de estos estudios son muy generalizados en cuanto al conocimiento 
de las materias primas empleadas, y a la infraestructura utilizada en el proceso de fabricación [2-5]. La presente investigación se ha realizado para tocar el tema de las materias primas y del proceso de conformado por extrusión mas a fondo, ya que no existe documentación clara al respecto en la región.

\section{Materiales y métodos}

La investigación se realizó en la Zona metropolitana de San José de Cúcuta, la población sobre la que se hizo el estudio fueron los materiales arcillosos pertenecientes a las formaciones geológicas León y Guayabo que afloran en esta zona. El muestreo realizado fue de tipo intencionado, se tomaron muestras del grupo Guayabo en la vereda la Alejandra, jurisdicción del municipio del El Zulia y muestras en la zona de los Vados jurisdicción del municipio de Los Patios; las muestras de la formación León se tomaron de la zona cercana al cerro Tasajero perteneciente al municipio de Cúcuta. La investigación realizada es de tipo exploratoria y experimental la cual se llevó a cabo en el laboratorio de molienda y moldeo, del Centro de Investigación de Materiales Cerámicos CIMAC.

El proceso de extrusión se realizó usando extrusora de laboratorio con vacío modelo NEW WAVE fabricada por la empresa Metal Souza Ltda., un molino de martillos de laboratorio marca Servitech modelo CT-
058, estufa de secado, vibrotamiz, juego de tamices, equipamiento para realizar prueba de hidrómetro, pinza amperimetrica marca Elmaleh referencia DT266C, plasticimetro de Pfefferkorn, horno mufla, balanzas digitales y un crometro Gabrielli para el establecimiento de la resistencia a la flexión.

A las muestras recolectadas se les realizó ensayos para determinar las características principales de las materias primas que entran al proceso de extrusión. El primero fue el índice de plasticidad, el cual se realizó utilizando el método de Pfefferkorn, luego se realizaron ensayos para determinar el tamaño de partícula, los cuales se llevaron a cabo utilizando la técnica del hidrómetro y el análisis por tamizado [6-8].

Posteriormente se procedió a la preparación de varias mezclas para ser usadas en el equipo de extrusión, las variables de trabajo en dichas mezclas fueron el contenido de arena y la variación del tamaño de grano respecto al patrón original extraído de la mina, la molienda se realizó por vía seca utilizando un molino de martillos de laboratorio. Una vez definidas las mezclas se procedió a realizar los diferentes ensayos en la extrusora de laboratorio, se utilizó una boquilla que da la forma de una baldosa de 4,0 centímetros de lado, se establecieron los tiempos de extrusión, la potencia requerida en términos de amperaje con respecto a una alimentación de 220 VAC y las características superficiales del

Figura 1. Fotografías digitales, a) proceso de extrusión de las muestras y b) probetas o muestras cocidas.

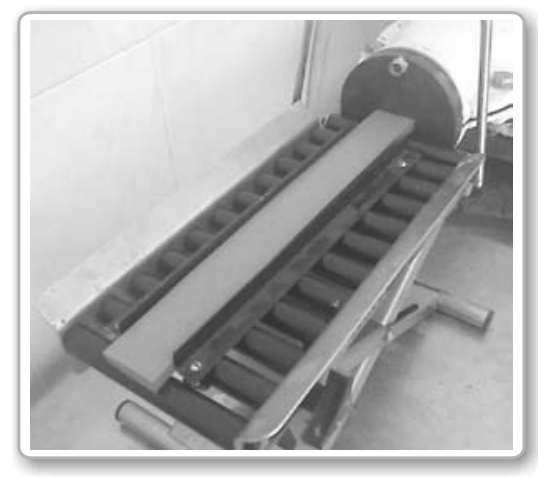

a)

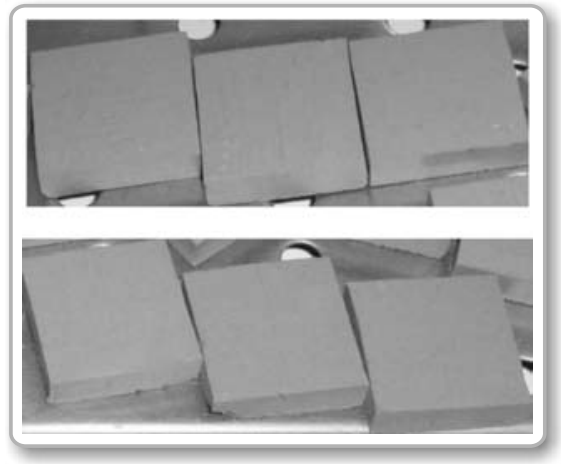

b) 
producto conformado. Terminado el proceso de extrusión, las probetas se llevaron a la estufa de secado por un tiempo de 24 horas a una temperatura de $110^{\circ} \mathrm{C}$, posteriormente al horno mufla a una temperatura de $1000^{\circ} \mathrm{C}$ durante 6 horas, finalmente las probetas fueron sometidas a los ensayos de absorción de agua (método de inmersión) y resistencia a la flexión. En la figura 1, se presentan fotografías del proceso de extrusión y de las probetas o muestras finales. Los ensayos realizados se llevaron a cabo de acuerdo a lo establecido en las normas técnicas colombianas para este tipo de materiales [9-13].

\section{Resultados y discusión}

Los expertos en cerámica afirman que para tener un buen resultado en la etapa de extrusión se debe considerar dos factores, el primero se refiere a la maquinaria o equipo utilizado para el moldeo y el segundo hace referencia a la materia prima [14].

En la tabla 1, se presentan los resultados del tamaño de partícula que permiten determinar la aptitud cerámica de las muestras, los cuales al graficarse en el triángulo de Winkler (ver figura 2), determina la zona especifica (Z1, Z2, Z3 o Z4) en la cual se encuentran las muestras estudiadas. De los resultados anteriores, se evidencia que ninguna de las muestras está dentro de una zona especifica, la zona mas cercana es Z3, cuya aptitud es para la fabricación de tejas, ladrillos perforados y pisos, sin embargo habría que aumentar el contenido de partículas superiores a $20 \mu \mathrm{m}$, es decir la fracción arenosa, para que las muestras se ubiquen en esta zona. De otra forma se aprecia que la muestra perteneciente a la formación León, tiene un mayor contenido de partículas finas (\% limos y \% arcilla), aportando mayor superficie específica a las partículas, contribuyendo en el aumento de la plasticidad, lo anterior se infiere de los resultados reportados en la tabla 2 , donde se observa un IP promedio de $24,5 \%$ para las muestras de la formación Guayabo, mientras que el IP para la formación León reportó un valor de $29,5 \%$.

Tabla 1. Porcentaje de arena, limos y arcillas según el tamaño de partícula de las muestras.

\begin{tabular}{|c|c|c|c|}
\hline \multirow{2}{*}{ MUESTRA } & \multicolumn{3}{|c|}{ TAMAÑO DE PARTICULA MÉTODO } \\
& \multicolumn{3}{|c|}{ HIDROMETRO } \\
\cline { 2 - 4 } & \%ARENAS & \%LIMOS & \%ARCILLA \\
\hline Grupo Guayabo, Municipio del El Zulia & 12,3 & 27,2 & 60,5 \\
\hline Grupo Guayabo, Municipio de Los Patios & 15,4 & 23,1 & 61,5 \\
\hline Formación León, Municipio Cúcuta & 4,5 & 33,9 & 61,6 \\
\hline
\end{tabular}

Figura 2. Ubicación de las muestras de arcilla en el triángulo de Winkler, usando los datos de la tabla 1.

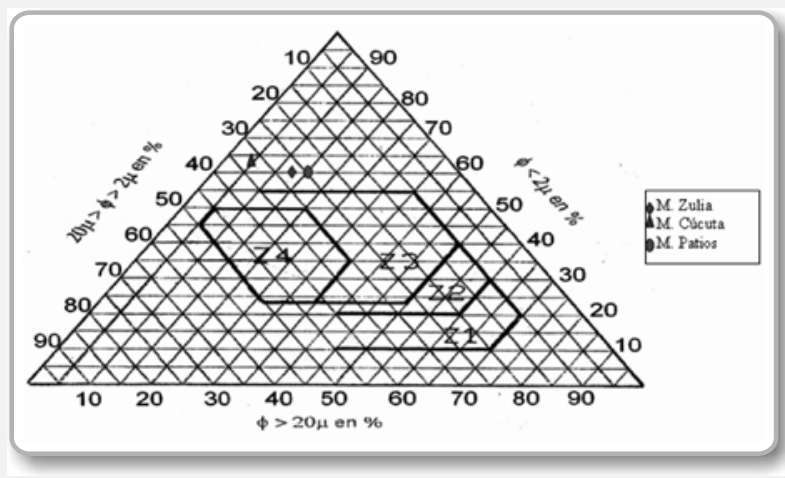

Tabla 2. Índices de plasticidad de las muestras.

\begin{tabular}{|l|c|}
\hline \multicolumn{1}{|c|}{ TIPO DE MUESTRA } & $\begin{array}{c}\text { INDICE DE PLASTICIDAD } \\
\text { (IP)* }\end{array}$ \\
\hline Grupo Guayabo, Municipio del El Zulia & $24,0 \%$ \\
\hline Grupo Guayabo, Municipio de Los Patios & $25,0 \%$ \\
\hline Formación León, Municipio Cúcuta & $29,5 \%$ \\
\hline
\end{tabular}

* Usando el método de Pfefferkorn

Los análisis de granulometría por tamizado complementaron los resultados del ensayo de análisis de partícula, se evidenció que en los diferentes tipos de arcillas (molida en molino de martillos), alrededor del $55 \%$ de la muestra eran pasante tamiz $120(125 \mu \mathrm{m})$, lo cual deja ver que existe una buena proporción entre las partículas gruesas y finas.

El comportamiento de los materiales arcillosos en el proceso de extrusión, se describe mediante la velocidad y corriente de extrusión, los cuales se presentan en la tabla 3. Se observa que la corriente promedio no varió, mientras que la velocidad oscilo entre 1,1 y $1,5 \mathrm{~cm} / \mathrm{s}$. 
Tabla 3. Comportamiento de los materiales arcillosos de la formación León y Guayabo en el proceso de conformado por extrusión.

\begin{tabular}{|c|c|c|c|}
\hline Muestra & $\begin{array}{c}\text { Velocidad } \\
(\mathbf{c m} / \mathbf{s})\end{array}$ & $\begin{array}{c}\text { Corriente } \\
\text { promedio (A)* }\end{array}$ & Observaciones del proceso \\
\hline Muestra Zulia & 1,5 & 4,2 & $\begin{array}{c}\text { Buen aspecto en bordes, } \\
\text { superficie lisa, }\end{array}$ \\
\hline Muestra Patios & 1,1 & 4,3 & $\begin{array}{c}\text { Superficie lisa, pequeños } \\
\text { defectos en bordes }\end{array}$ \\
\hline Muestra Cúcuta & 1,2 & 4,3 & $\begin{array}{c}\text { Se presenta algunos defectos } \\
\text { en la superficie de la pieza }\end{array}$ \\
\hline
\end{tabular}

* En el momento en que ya existe conformado de la pieza, en este instante se ha estabilizado el consumo eléctrico.

La muestra proveniente del municipio de El Zulia presentó la mejor valoración en todos los aspectos, es evidente que el índice de plasticidad más bajo de esta arcilla influyó este resultado. Las probetas elaboradas con la arcilla de la formación León presentaron defectos en su superficie, al realizar nuevas pruebas disminuyendo el porcentaje de limos se logró disminuir este inconveniente, el rango de mejor comportamiento para este material arcilloso estuvo entre el $22 \%$ y el $28 \%$ de fracción limosa.

Las probetas obtenidas se llevaron a la estufa de secado y posteriormente al horno mufla a una temperatura de $1000{ }^{\circ} \mathrm{C}$, a las probetas sinterizadas se les realizó los ensayos de absorción de agua y resistencia a la flexión, dichos resultados se presentan en la tabla 4 , donde se aprecia que hay una variación aproximada del 10\% entre los valores encontrados para estos parámetros.

Tabla 4. Absorción de agua y resistencia a la flexión de las probetas elaboradas

\begin{tabular}{|c|c|c|}
\hline Muestra & \% Absorción de agua & $\begin{array}{c}\text { Resistencia a la Flexión } \\
\text { Promedio (Newton/mm }\end{array}$ \\
\hline Muestra Zulia & 9,7 & 18,2 \\
\hline Muestra Patios & 10,5 & 19,5 \\
\hline Muestra Cúcuta & 10,5 & 21,0 \\
\hline
\end{tabular}

En miras de encontrar las condiciones más óptimas que debe presentar el material a utilizar en el proceso de extrusión se elaboraron varias mezclas a las cuales se les realizó cambios en el tamaño de grano y en el contenido de arena. Las características de las diferentes mezclas elaboradas se presentan en la tabla 5 .

Tabla 5. Descripción de las mezclas preparadas

\begin{tabular}{|c|c|}
\hline Código & Características de la mezcla \\
\hline VG1 & $70 \%$ de pasante malla 60 y $30 \%$ de retenido malla 60 \\
\hline VG2 & $60 \%$ de pasante malla 20 y $40 \%$ de retenido malla 20 \\
\hline VG3 & $70 \%$ pasante malla 60 y $30 \%$ pasante malla 20 \\
\hline VG4 & $60 \%$ pasante malla 60 y $40 \%$ de retenido malla 20 \\
\hline VA1 & $90 \%$ de material arcilloso y $10 \%$ de material arenoso( en peso) \\
\hline VA2 & $80 \%$ de material arcilloso y $20 \%$ de material arenoso( en peso) \\
\hline
\end{tabular}

Las mezclas VG se realizaron para determinar la influencia en la variación de las proporciones de los granos grandes y pequeños, para esto se utilizó el material arcilloso de la zona de El Zulia. A las probetas VG sólo se le realizó un análisis visual en la operación de extrusión, las muestras sinterizadas a $1000{ }^{\circ} \mathrm{C}$ fueron sometidas a los ensayos de absorción de agua y resistencia a la flexión, las mezclas VA se realizaron para determinar el contenido de arenas más adecuado para realizar un producto con buenas condiciones técnicas y con el menor consumo eléctrico. Los resultados obtenidos se presentan en las tablas 6 y 7 , donde se observa, que la muestra que presenta mayor dificultad para fluir, es la que tiene la menor resistencia a la flexión. Para las muestras, donde se varió la concentración de arena (VA1, VA2 y VA3), se aprecia que a medida que aumenta el contenido de arena, el índice de plasticidad (IP) y la resistencia a la flexión disminuye, mientras que el porcentaje de absorción de agua permanece constante.

Tabla 6. Comportamiento de las probetas sometidas a cambios de granulometría

\begin{tabular}{|c|l|c|c|}
\hline Muestra & Características en extrusión & $\begin{array}{c}\text { \% absorción } \\
\text { de agua }\end{array}$ & $\begin{array}{c}\text { Resistencia } \\
\text { a la flexión } \\
\text { (Newton/mm }\end{array}$ \\
\hline VG1 & $\begin{array}{l}\text { Buena fluidez en la extrusora, } \\
\text { dificultad en el corte, aspecto } \\
\text { superficial bueno. }\end{array}$ & 10,2 & 20,0 \\
\hline VG2 & Buena fluidez, superficie lisa, & 9,9 & 19,8 \\
\hline VG3 & Buena fluidez, superficie lisa & 10,3 & 19,8 \\
\hline VG4 & $\begin{array}{l}\text { Dificultad para fluir, presenta } \\
\text { problemas en la superficie }\end{array}$ & 10,4 & 16,5 \\
\hline
\end{tabular}


Tabla 7. Comportamiento de probetas sometidas a variación del contenido de arena

\begin{tabular}{|c|c|c|c|c|c|}
\hline Muestra & IP (\%) & $\begin{array}{c}\text { Velocidad } \\
\text { (cm/s) }\end{array}$ & $\begin{array}{c}\text { Amperaje } \\
\text { promedio* }\end{array}$ & $\begin{array}{c}\text { \% absorción } \\
\text { de agua* }\end{array}$ & $\begin{array}{c}\text { Resistencia a } \\
\text { la flexión }^{*} \\
\text { (Newton/mm }^{\text {( }) ~}\end{array}$ \\
\hline VA0 & 25,0 & 1,1 & 4,3 & 10,5 & 19,5 \\
\hline VA1 & 22,5 & 1,7 & 4,2 & 10,4 & 17,2 \\
\hline VA2 & 21,6 & 1,8 & 4,3 & 10,5 & 16,5 \\
\hline VA3 & 19,0 & 1,1 & 5,0 & 10,4 & 11,2 \\
\hline
\end{tabular}

* Temperatura de cocción $1000^{\circ} \mathrm{C}$

Aunque los resultados de las pruebas de absorción de agua, y resistencia a la flexión están en función de variables como la mineralogía de la arcilla, la temperatura de cocción, la composición química y la distribución granulométrica entre otras, en la extrusión éstas juegan un papel importante, ya que al conseguir una buena compactación de la pieza extruida se logra disminuir la porosidad de la misma, influyendo positivamente en las propiedades mecánicas de la muestra. Los resultados de la tabla 7 , dejan ver que los valores óptimos se dan para las mezclas VA1 y VA2. La resistencia a la flexión permite establecer que el aumento del contenido del material arenoso disminuye el valor de esta propiedad, lo cual trae como consecuencia que haya un mayor consumo energético cuando se trabaja con valores elevados de arena, ya que se requiere una mayor temperatura de cocción para alcanzar un resultado como el de la muestra sin agregar arena (VO).

\section{Conclusiones}

De acuerdo a los resultados obtenidos, la aptitud cerámica de las arcillas de la región según el triángulo de Winkler está muy cerca de la zona Z3, cuyo uso es para la fabricación de tejas, ladrillos perforados y pisos.

El diseño de la máquina y la materia prima son los dos factores principales a los que hay que prestar atención si se quiere hacer un buen producto cerámico utilizando el moldeo por extrusión.

El estudio de las arcillas utilizadas permitió establecer que las arcillas con una granulometría variada son las más adecuadas para realizar un buen moldeo y obtener un nivel de compactación óptimo.

Las arcillas con gran cantidad de partículas finas no permiten que haya una buena compactación de la pieza, presentan problemas para el corte, reducen el buen aspecto superficial de la pieza y la resistencia a la flexión puede verse reducida si se forman laminaciones o corazón negro en el moldeo de la baldosa. Las arcillas con alto contenido de partículas gruesas presentan resultados similares a los expuestos para las partículas finas.

Lo ensayos realizados a las mezclas con variación de material arenoso ha permitido establecer que los rangos más adecuados para la formulación de las pastas cerámicas en la zona metropolitana de Cúcuta deben estar en: la fracción arenosa entre 20 y $27 \%, 23$ y $28 \%$ para la fracción de limos y entre 47 y $58 \%$ para la fracción arcillosa; el índice de plasticidad debe estar cercano al 22\%. Las formulaciones establecidas en estos rangos permiten obtener buenas velocidades de extrusión, consumos energéticos adecuados y una buena calidad del producto fabricado.

\section{Agradecimientos}

Los autores agradecen al departamento Administrativo de Ciencia, Tecnología e Innovación COLCIENCIAS a través del programa Jóvenes Investigadores P-2007-027 y al Centro de Investigación de Materiales Cerámicos CIMAC por el apoyo brindado.

\section{Bibliografía}

\section{[1]. CARRILLO CÁRDENAS, Mónica} y AREVALO MONROY, Iraní. Actualización de la información y puesta en marcha de una aplicación sistematizada para el sector cerámico del área metropolitana de san José de Cúcuta. Plan de estudios de Ingeniería de Producción Industrial. San José de Cúcuta. Universidad Francisco de Paula Santander. Facultad de Ingeniería. Año 2008. 
[2]. PRATO CRUZ Elcy Johanna. Planteamiento de modelos productivos para la estandarización de los procesos de producción de las Mipymes del sector cerámico del área metropolitana de san José de Cúcuta. Grupo de investigación en tecnología cerámica GITEC. Universidad Francisco de Paula Santander. 2007.

[3]. SANCHEZ Jennifer Y PEÑARANDA Claudia. Estudio de las etapas de extrusión, secado y cocción, del sistema productivo de la línea Monserrate para mejorar su productividad en la empresa Cerámica Andina Ltda. Trabajo de grado Ingeniería de Producción Industrial, San José de Cúcuta. Universidad Francisco de Paula Santander, Facultad de ingenierías. Año 2005.

[4]. SAYAGO CLAVIJO Elluz Johanna. Estudio de los defectos mayores de las tabletas vitrificadas en la ladrillera Casablanca. Trabajo de grado Ingeniería de Producción Industrial, San José de Cúcuta. Universidad Francisco de Paula Santander, Facultad de ingenierías. Año 2006.

[5]. JESKAR LTDA Inventario geológico minero, ambiental, tecnológico y empresarial de los minerales no energéticos del Norte de Santander, San José de Cúcuta, junio de 2000.

[6]. INSTITUTO DE TECNOlOGIA CERAMICA. Manual para el control de la calidad de materias primas arcillosas. ITC, 2004. 168 Pág.

[7]. INSTITUTO DE TECNOLOGIA CERAMICA. Materias primas para la fabricación de soportes de baldosas cerámicas. Castellón-España. Segunda edición, ITC, 2002. 291 Pág.

[8]. INSTITUTO DE TECNOLOGIA CERAMICA. Manual para el control de la calidad de materias primas empleadas

en la preparación de los vidriados cerámicos. Castellón-España., ITC, 2000. 320 Pág.

[9]. INSTITUTO COLOMBIANO DE NORMAS TECNICAS Y CERTIFICACION, NTC4017 Métodos para muestreo y ensayos de unidades de mampostería y otros productos de arcilla. Bogota. ICONTEC. 2005

[10]. INSTITUTO COLOMBIANO DE NORMAS TECNICAS Y CERTIFICACION, NTC4205 unidades de mampostería de arcilla cocida. Ladrillos y bloques cerámicos. Bogota D.C. ICONTEC, primera actualización. 2005.

[11]. INSTITUTO COLOMBIANO DE NORMAS TECNICAS Y CERTIFICACION, NTC 919 baldosas cerámicas. Definiciones, clasificación, características y rotulado. Bogota D.C. ICONTEC, cuarta actualización. 2005.

[12]. INSTITUTO COLOMBIANO DE NORMAS TECNICAS Y CERTIFICACION, NTC 4321-3 baldosas cerámicas parte 3. Método de ensayo para determinar la absorción de agua, porosidad aparente, densidad relativa aparente y densidad aparente. Bogota D.C. ICONTEC, 2005.

[13]. INSTITUTO COLOMBIANO DE NORMAS TECNICAS Y CERTIFICACION, NTC 4321-4 baldosas cerámicas parte 4. Método de ensayo para determinar el modulo de rotura y la resistencia a la flexión. Bogota D.C. ICONTEC, 2005.

[14]. FERNÁNDEZ ABAJO MARCELINO, Manual sobre fabricación de baldosas, tejas y ladrillos [CD-ROM]. Laboratorio Técnico Cerámico S.L. AÑO 2000. $370 \mathrm{p}$. 